\title{
Low Effective Electron Mass InGaAs/InAlAs for High Power Terahertz Quantum Cascade Lasers
}

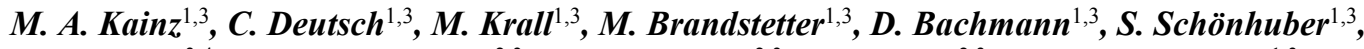 \\ H. Detz ${ }^{3,4}$, D. C. MacFarland ${ }^{2,3}$, A. M. Andrews ${ }^{2,3}$, G. Strasser ${ }^{2,3}$, and K. Unterrainer ${ }^{1,3}$ \\ 1. Photonics Institute, TU Wien, Gusshausstraße 27-29, A-1040 Vienna, Austria \\ 2. Institute of Solid State Electronics, TU Wien, Floragasse 7, A-1040 Vienna, Austria \\ 3. Center for Micro- and Nanostructures, TU Wien, Floragasse 7, A-1040 Vienna, Austria \\ 4. Austrian Academy of Sciences, Dr. Ignaz Seipel-Platz, 2, A-1010 Vienna, Austria
}

Quantum cascade lasers (QCLs) are powerful sources of coherent radiation covering the frequency range from mid-infrared to terahertz. In the terahertz frequency range the active region is normally realized using a $\mathrm{GaAs} / \mathrm{Al}_{\mathrm{x}} \mathrm{Ga}_{1-\mathrm{x}} \mathrm{As}$ semiconductor heterostructure. This material system enables a variable conduction band offset by changing the Al-content in the barrier layers without introducing a significant lattice mismatch between the barrier and well material. In comparison to the standard GaAs-based material system, active regions based on material systems with a lower effective electron mass are highly beneficial for the design of terahertz QCLs as the optical gain increases for a lower effective electron mass [1]. Promising material systems are based on InGaAs or InAs with an effective electron mass of 0.043 and 0.023 , respectively, compared to that of GaAs $(0.067)[2,3]$.

In this work we present a systematic study of growth related asymmetries for terahertz QCLs based on the InGaAs/InAlAs material system lattice matched on InP. A nominally symmetric active region enables the comparison of the positive and negative bias direction of the very same device [4]. With such bias dependent performance measurements asymmetries like dopant migration and interface roughness, which play a crucial role in this material system, are studied and result in a preferred electron flow in growth direction. A structure based on a three well optical phonon depletion scheme is optimized for this bias direction. Depending on the doping concentration the performance of the QCLs shows a trade-off between maximum operating temperatures and high output powers. While a peak output power of $151 \mathrm{~mW}$ is achieved for a sheet doping density of $7.3 \times 10^{10} \mathrm{~cm}^{-2}$, the highest operation temperature of $155 \mathrm{~K}$ is found for $2 \times 10^{10} \mathrm{~cm}^{-2}$. By further attaching a hyperhemispherical GaAs lens to a laser facet, the peak output power could be improved and reaches a record output power for double metal waveguide terahertz QCLs of almost $600 \mathrm{~mW}$.
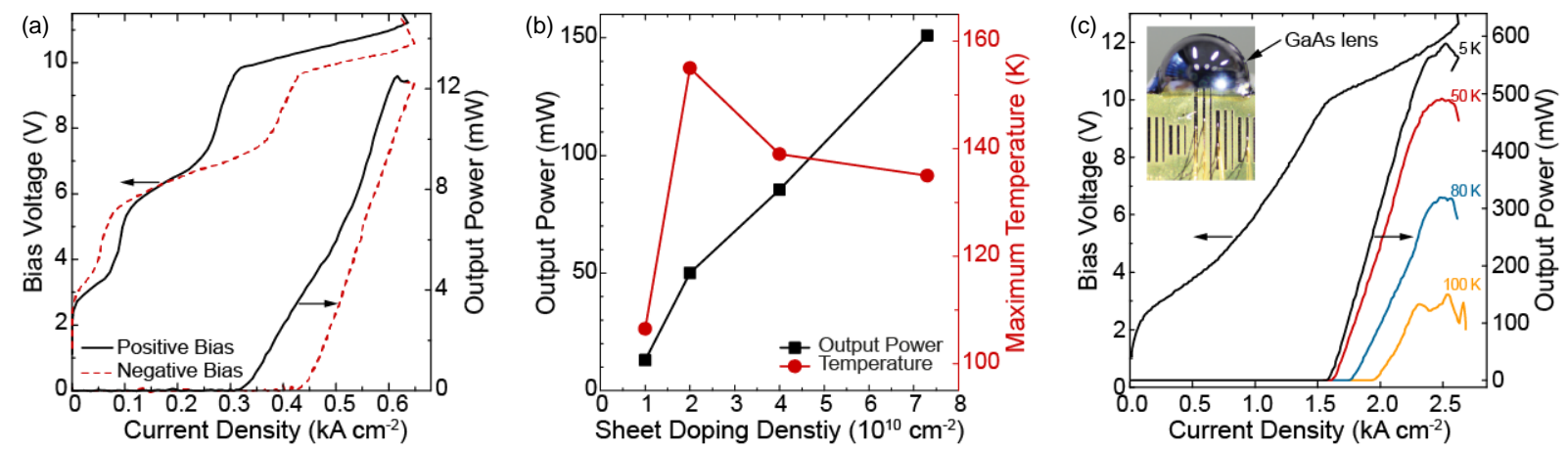

Fig. 1 (a) Light-current-voltage measurement at $5 \mathrm{~K}$ for both bias directions of the symmetric active region design showing a higher slope efficiency for the negative bias polarity. (b) Trade-off between high output powers and the maximum operation temperature as a function of the sheet doping density. (c) Improved out-coupling efficiency with an attached hyperhemispherical GaAs lens yields a record output power of $587 \mathrm{~mW}$ for double metal waveguide terahertz QCLs.

\section{References}

[1] E. Benveniste, A. Vasanelli, A. Delteil, J. Devenson, R. Teissier, A. Baranov, A. M. Andrews, G. Strasser, I. Sagnes, and C. Sirtori, "Influence of the material parameters on quantum cascade devices," Appl. Phys. Lett. 93, 131108 (2008).

[2] M. Fischer, G. Scalari, Ch. Walther, J. Faist, "Terahertz quantum cascade lasers based on $\operatorname{In}_{0.53} \mathrm{Ga}_{0.47} \mathrm{As} / \mathrm{In}_{0.52} \mathrm{Al}_{0.48} \mathrm{As} / \mathrm{InP}$," J. Cryst. Growth 311, 1939 (2009).

[3] M. Brandstetter, M. A. Kainz, T. Zederbauer, M. Krall, S. Schönhuber, H. Detz, W. Schrenk, A. M. Andrews, G. Strasser, and K. Unterrainer, "InAs based terahertz quantum cascade lasers," Appl. Phys. Lett. 108, 11109 (2016)

[4] C. Deutsch, H. Detz, M. Krall, M. Brandstetter, T. Zederbauer, A. M. Andrews, W. Schrenk, G. Strasser, and K. Unterrainer, "Dopant migration effects in terahertz quantum cascade lasers," Appl. Phys. Lett. 102, 201102 (2013). 
(C) ( 2017 IEEE. Personal use of this material is permitted. Permission from IEEE must be obtained for all other uses, in any current or future media, including reprinting/republishing this material for advertising or promotional purposes, creating new collective works, for resale or redistribution to servers or lists, or reuse of any copyrighted component of this work in other works.

This is the accepted version of M. Kainz et al., "Low Effective Electron Mass InGaAs/InAIAs for High Power Terahertz Quantum Cascade Lasers," 2017 Conference on Lasers and Electro-Optics Europe \& European Quantum Electronics Conference (CLEO/Europe-EQEC), Munich, 2017, doi :

10.1109/CLEOE-EQEC.2017.8086429 